\title{
Fitossociologia de uma floresta de restinga no Parque Estadual Paulo César Vinha, Setiba, município de Guarapari (ES)
}

\author{
ANDRÉ M. DE ASSIS ${ }^{1,4}$, OBERDAN J. PEREIRA² e LUCIANA D. THOMAZ ${ }^{3}$
}

(recebido: 27 de novembro de 2002; aceito: 19 de fevereiro de 2004)

\begin{abstract}
Phytosociology of a restinga forest in the "Paulo César Vinha" State Park, Setiba, Guarapari (Espírito Santo)). The vegetation structure of a restinga forest in the "Paulo César Vinha" State Park (PCVSP), Setiba, Municipality of Guarapari (ES) was studied using 100 sampling plots $(10 \times 10 \mathrm{~m})$ in four transects perpendicular to the coastline. A total of 2,106 trees (dbh $\geq 4.8 \mathrm{~cm}$ ) were sampled and total basal area was $27.52 \mathrm{~m}^{2} . \mathrm{ha}^{-1}$ and species diversity $\left(\mathrm{H}^{\prime}\right)$ was 3.73 nats $(67$ dead standing trees were not included). Based on decreasing importance values (VI), the following families and species outstanded: Myrtaceae, Sapotaceae, Annonaceae, Bombacaceae, and Meliaceae; Pouteria coelomatica, Myrciaria floribunda, Oxandra nitida, Chrysophyllum lucentifolium and Aspidosperma parvifolium. Forest structure and species composition vary on the gradient from the ocean landward, forming three dissimilar species groups. Some species were found only in one of these groups and species dominance also differed zonally. It is proposed that the studied community is called "non-flooded forest formation" instead of the traditionally "Myrtaceae forest" or "dry forest", since family and species composition do not allow a more detailed classification.
\end{abstract}

Key words - phytosociology, forest formation, restinga

RESUMO - (Fitossociologia de uma floresta de restinga no Parque Estadual Paulo César Vinha, Setiba, município de Guarapari (ES)). A vegetação de um trecho da floresta de restinga no Parque Estadual Paulo César Vinha (PEPCV), Setiba, município de Guarapari (ES) foi amostrada através de 100 parcelas de $10 \times 10 \mathrm{~m}$ cada, plotadas em quatro linhas perpendiculares ao mar. Foram inventariados 2.106 indivíduos (DAP $\geq 4,8 \mathrm{~cm}$ ), fornecendo uma área basal de 27,52 $\mathrm{m}^{2}$.ha-1 e diversidade (H') de 3,73 nats, excetuando os 67 mortos em pé. Segundo valores decrescentes de importância (VI), destacaram-se as famílias Myrtaceae, Sapotaceae, Annonaceae, Bombacaceae e Meliaceae, e as espécies Pouteria coelomatica, Myrciaria floribunda, Oxandra nitida, Chrysophyllum lucentifolium e Aspidosperma parvifolium. A floresta apresenta variações estruturais e florísticas no gradiente mar-continente, com formação de três grupos dissimilares de espécies. Ocorrência exclusiva de espécies nos grupos e diferenças entre os principais táxons de cada grupo, evidenciam uma zonação. Propõe-se o termo geral "formação florestal não inundável" para esta comunidade, frente aos tradicionais "Mata de Myrtaceae" e "Mata Seca", uma vez que a composição de suas principais famílias e espécies não permite separá-las de acordo com os critérios de classificação destas comunidades.

Palavras-chave - fitossociologia, formação florestal, restinga

\section{Introdução}

Diferentes comunidades vegetais podem ser encontradas ao longo da costa brasileira, em função das condições climáticas e edáficas e de fatores temporais de caráter sucessional (Araujo 1987). Essas comunidades podem ser denominadas vegetação de restinga, embora o termo "restinga" também apresente outros significados (Suguio \& Tessler 1984).

Dentre as formações vegetais existentes nas restingas estão as comunidades florestais, que podem ou não sofrer inundações durante o ano (Araujo \&

1. Museu de Biologia Prof. Mello Leitão, Av. José Ruschi 4, 29650-000 Santa Teresa, ES, Brasil.

2. FAESA, Ciências Biológicas, Rod. Serafim Derenze 33115, 29048-450 Vitória, ES, Brasil.

3. Universidade Federal do Espírito Santo, Setor Botânica, Av. Fernando Ferrari s/n, 29060-900 Vitória, ES, Brasil.

4. Autor para correspondência: pouteria@terra.com.br
Henriques 1984, Silva 1998); apresentam fisionomia, estrutura e composição florística diferenciadas, e recebem variadas denominações como mata seca, mata de Myrtaceae (Araujo \& Henriques 1984, Pereira 1990, Bastos 1996), mata arenosa (Waechter 1985), floresta arenícola costeira (Trindade 1991), floresta arenosa litorânea (Fabris 1995) ou simplesmente mata ou floresta de restinga (Silva at al. 1994, Sugyiama \& Mantovani 1994, César \& Monteiro 1995, Lobão \& Kurtz 2000).

Segundo Araujo (1992), a ausência de dados ecológicos e fisionômicos de diversos trechos do litoral brasileiro e a falta de consenso sobre o que constitui a vegetação sobre as planícies costeiras arenosa são as maiores dificuldades na determinação de um sistema de classificação dos tipos vegetacionais que seja adequado para toda a costa brasileira.

Estudos nas restingas do Estado do Espírito Santo, com enfoque na fitossociologia de formações florestais, limitam-se ao de Fabris (1995) em Setiba, Guarapari. 
Pereira (1990) descreveu para esta restinga diferentes formações vegetais, dentre elas a mata de Myrtaceae e a mata seca, com informações sobre sua fitofisionomia.

Neste trabalho foi analisado um trecho de floresta de restinga para descrição de sua estrutura fitossociológica, comparação com outras áreas da costa brasileira e identificação de agrupamentos de espécies, possibilitando rever a classificação desse tipo vegetacional.

\section{Material e métodos}

O Parque Estadual Paulo César Vinha (PEPCV) compreende uma planície litorânea de aproximadamente 1.500 ha em Setiba, município de Guarapari (Estado do Espírito Santo), entre as coordenadas $20^{\circ} 33^{\prime}-20^{\circ} 38^{\prime} \mathrm{S}$ e $40^{\circ} 23^{\prime}$ $40^{\circ} 26^{\prime}$ W. O clima da região é do tipo Aw, segundo classificação de Koeppen, apresentando temperatura média anual de $23,3{ }^{\circ} \mathrm{C}$, precipitação média anual de $1.307 \mathrm{~mm}$ e umidade relativa média anual de $80 \%$ (Fabris 1995).

O sedimento do trecho estudado, bem como de outras formações dessa unidade de conservação, é predominantemente arenoso (Pereira 1990, Fabris 1995), originado por deposição marinha no Holoceno, principalmente em função da variação do nível relativo do mar (Flexor et al. 1984).

A formação florestal analisada localiza-se aproximadamente a 150 metros da linha de maré alta, tendo como limite leste a comunidade arbustiva pós-praia e, à oeste, a aberta de Clusia, mais afastada do mar (Pereira 1990). No sentido norte-sul a floresta é praticamente contínua, sendo cortada apenas por algumas trilhas perpendiculares à linha de costa. A floresta apresenta fisionomias variadas para o interior do continente, relacionadas à altura e densidade dos indivíduos e composição florística, não havendo afloramento do lençol freático.

A análise quantitativa foi realizada no sentido marcontinente, com 100 parcelas de $10 \times 10 \mathrm{~m}$ distribuídas sobre quatro linhas perpendiculares à praia, espaçadas entre si por um intervalo de 35 metros. Em cada linha foram alocadas 25 parcelas contíguas. A amostragem incluiu indivíduos com perímetro à altura do peito $(\mathrm{PAP}) \geq 15 \mathrm{~cm}$, que foram plaqueteados com números seqüenciais para posterior identificação, estando os materiais testemunhos depositados no Herbário VIES, da Universidade Federal do Espírito Santo. A altura dos indivíduos foi estimada com auxílio de vara de poda com medida previamente conhecida. Os parâmetros fitossociológicos empregados foram frequência, densidade e dominâncias absolutas e relativas, valor de cobertura e de importância, segundo Mueller-Dombois \& Ellenberg (1974).

Para testar a ocorrência de variações na fisionomia da floresta em direção ao continente foi utilizada análise de similaridade florística, por meio do índice de Sørensen, reunindo os dados da parcela 1 de cada faixa de maneira a constituir uma unidade de $10 \times 40 \mathrm{~m}$, sendo esse procedimento repetido para as parcelas subseqüentes até a de número 25 , a mais afastada da linha de costa. O dendograma foi construído pelo agrupamento hierárquico aglomerativo ("weighted pairgroup method"). As análises fitossociológica e de similaridade foram realizadas no programa Fitopac 1 (Shepherd 1986).

\section{Resultados}

A floresta de restinga do PEPCV possui o estrato superior entre 6 e 10 metros, onde estão incluídos $68 \%$ dos indivíduos amostrados (figura 1). A altura média do trecho analisado foi 8,44 metros $( \pm 2,73)$, com indivíduos emergentes alcançando entre 18 e 20 metros, representados, dentre outras espécies, por Aspidosperma parvifolium, Buchenavia capitata, Eriotheca pentaphylla e Protium heptaphyllum. Os representantes de menor altura (3 e 4 metros) são, em sua maioria, do estrato inferior da floresta (sub-bosque), como Bactris vulgaris, Mollinedia glabra, Capparis flexuosa e Erythroxylum oxypetalum.

A variação diamétrica indicou poucos indivíduos de grande porte (figura 2), merecendo destaque neste aspecto Buchenavia capitata, Clusia hilariana, Coussapoa microcarpa e Syderoxylon obtusifolium. A maioria dos indivíduos está incluída na classe de diâmetro entre 10 e 15 centímetros, resultando em um diâmetro médio de 11,01 centímetros $( \pm 6,73)$.

Foram amostrados 2.106 indivíduos em 1 ha nessa floresta de restinga, que apresentou área basal de $27,52 \mathrm{~m}^{2} \cdot \mathrm{ha}^{-1}$, índice de diversidade de Shannon-Weaver de 3,73 nats e equabilidade (J) igual a 0,826 (tabela 1 ). Dentre os indivíduos amostrados, houveram 67 mortos

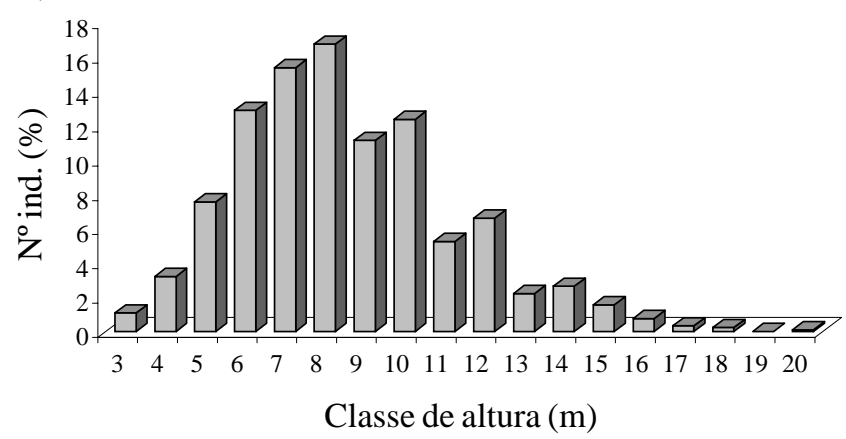

Figura 1. Distribuição da porcentagem do número de indivíduos por classe de diâmetro na floresta de restinga do PEPCV, Setiba, Guarapari, ES ( $\mathrm{n}=2106)$.

Figure 1. Distribution of percentage of the number of individuals by class of diameter in the restinga forest of the PEPCV, Setiba, Guarapari, ES $(n=2106)$. 


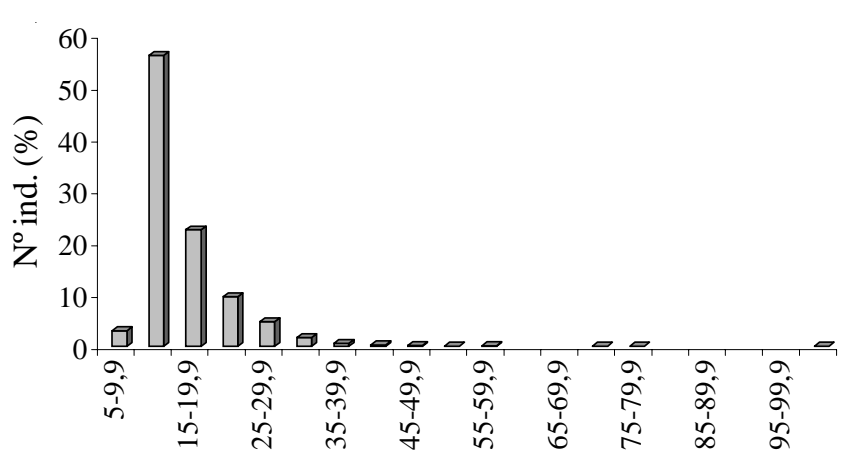

Classe de diâmetro $(\mathrm{cm})$

Figura 2. Distribuição do número de indivíduos por classe de altura na floresta de restinga do PEPCV, Setiba, Guarapari, ES $(n=2106)$.

Figure 2. Distribution of number of individuals by class of height in the restinga forest of the PEPCV, Setiba, Guarapari, ES $(n=2106)$. em 47 parcelas, totalizando uma área basal de $0,9 \mathrm{~m}^{2} \cdot \mathrm{ha}^{-1}$.

Dentre as 38 famílias encontradas neste levantamento (tabela 1), as Myrtaceae, com riqueza e densidade superiores às demais, ocupou a primeira colocação em valor de importância (VI), mesmo com Sapotaceae apresentando maior dominância (área basal). Famílias representadas por uma ou duas espécies, como Annonaceae, Bombacaceae, Apocynaceae e Burseraceae estão dentre aquelas com maiores VI, em função da elevada área basal e/ou densidade de seus indivíduos.

No levantamento fitossociológico foram identificados 92 táxons (tabela 2), sendo Pouteria coelomatica, Myrciaria floribunda e Oxandra nitida os principais em VI. Oxandra nitida apresentou maior densidade enquanto $M$. floribunda foi a mais freqüente; no entanto, a expressiva dominância de $P$. coelomatica

Tabela 1. Parâmetros fitossociológicos das famílias amostradas no PEPCV, Setiba, Guarapari, ES, em ordem decrescente de VI

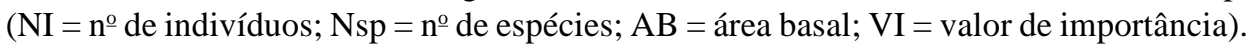

Table 1. Phytossociologic parameters of the families found at the PEPCV, Setiba, Guarapari, ES, ordered by decreasing VI $\left(\mathrm{NI}=\mathrm{n}^{\mathrm{o}}\right.$ of individuals; $\mathrm{Nsp}=\mathrm{n}^{\mathrm{o}}$ of species; $\mathrm{AB}=$ basal area; $\mathrm{VI}=$ value of importance $)$.

\begin{tabular}{rlrrrrrr}
\hline & Famílias & NI & Nsp & $\%$ sp & AB & VI & $\%$ V I \\
\hline 1. & Myrtaceae & 562 & 20 & 21,74 & 5,188 & 56,24 & 18,75 \\
2. & Sapotaceae & 350 & 9 & 9,78 & 5,256 & 46,20 & 15,40 \\
3. & Annonaceae & 216 & 2 & 2,17 & 1,896 & 25,34 & 8,45 \\
4. & Bombacaceae & 100 & 2 & 2,17 & 2,172 & 17,66 & 5,89 \\
5. & Meliaceae & 125 & 3 & 3,26 & 1,105 & 17,37 & 5,79 \\
6. & Apocynaceae & 76 & 2 & 2,17 & 0,959 & 12,12 & 4,04 \\
7. & Lauraceae & 57 & 5 & 5,43 & 1,206 & 11,56 & 3,85 \\
8. & Nyctaginaceae & 60 & 2 & 2,17 & 1,103 & 11,33 & 3,78 \\
9. & Burseraceae & 44 & 1 & 1,09 & 1,373 & 10,57 & 3,52 \\
10. & Fabaceae & 64 & 3 & 3,26 & 0,482 & 9,37 & 3,12 \\
11. & Thymelaceae & 58 & 1 & 1,09 & 0,526 & 8,81 & 2,94 \\
12. & Simaroubaceae & 48 & 2 & 2,17 & 0,637 & 8,09 & 2,70 \\
13. & Moraceae & 17 & 5 & 5,43 & 1,190 & 6,55 & 2,18 \\
14. & Olacaceae & 32 & 3 & 3,26 & 0,459 & 6,46 & 2,15 \\
15. & Clusiaceae & 33 & 4 & 4,35 & 0,752 & 6,37 & 2,12 \\
16. & Rubiaceae & 43 & 2 & 2,17 & 0,263 & 5,95 & 1,98 \\
17. & Capparaceae & 36 & 2 & 2,17 & 0,508 & 5,85 & 1,95 \\
18. & Sapindaceae & 26 & 3 & 3,26 & 0,257 & 4,68 & 1,56 \\
19. & Cactaceae & 1 & 1,09 & 0,331 & 4,46 & 1,49 \\
20. & Malpighiaceae & 25 & 1 & 1,09 & 0,330 & 4,05 & 1,35 \\
21. & Mimosaceae & 21 & 1 & 1,09 & 0,277 & 3,75 & 1,25 \\
22. & Myrsinaceae & 21 & 1 & 1,09 & 0,398 & 3,52 & 1,17 \\
23. & Polygonaceae & 16 & 1 & 1,09 & 0,125 & 2,21 & 0,74 \\
24. & Arecaceae & 14 & 1 & 1,09 & 0,024 & 1,37 & 0,46 \\
25. & Erythroxylaceae & 2 & 2,17 & 0,043 & 1,35 & 0,45 \\
26. & Bignoniaceae & 9 & 1 & 1,09 & 0,132 & 1,11 & 0,37 \\
& & 4 & & & & & continua
\end{tabular}


continuação

\begin{tabular}{|c|c|c|c|c|c|c|c|}
\hline & Famílias & NI & Nsp & $\% \mathrm{sp}$ & $\mathrm{AB}$ & VI & $\% \mathrm{VI}$ \\
\hline 27. & Caesalpiniaceae & 4 & 1 & 1,09 & 0,114 & 1,04 & 0,35 \\
\hline 28. & Ebenaceae & 6 & 1 & 1,09 & 0,033 & 0,95 & 0,32 \\
\hline 29. & Combretaceae & 1 & 1 & 1,09 & 0,214 & 0,93 & 0,31 \\
\hline 30. & Ochnaceae & 6 & 1 & 1,09 & 0,019 & 0,90 & 0,30 \\
\hline 31. & Solanaceae & 5 & 1 & 1,09 & 0,019 & 0,85 & 0,28 \\
\hline 32. & Oleaceae & 5 & 1 & 1,09 & 0,016 & 0,84 & 0,28 \\
\hline 33. & Euphorbiaceae & 3 & 1 & 1,09 & 0,068 & 0,72 & 0,24 \\
\hline 34. & Monimiaceae & 3 & 1 & 1,09 & 0,018 & 0,53 & 0,18 \\
\hline 35. & Melastomataceae & 2 & 1 & 1,09 & 0,009 & 0,34 & 0,11 \\
\hline 36. & Anacardiaceae & 1 & 1 & 1,09 & 0,014 & 0,21 & 0,07 \\
\hline 37. & Celastraceae & 1 & 1 & 1,09 & 0,005 & 0,17 & 0,06 \\
\hline 38. & Rutaceae & 1 & 1 & 1,09 & 0,002 & 0,16 & 0,05 \\
\hline
\end{tabular}

garantiu a sua primeira colocação. Dentre as 92 espécies amostradas, algumas se destacaram pelos altos valores de dominância como Protium heptaphyllum, Pseudobombax grandiflorum, Coussapoa microcarpa e Pouteria sp., estando, também, entre as de maior altura.

O dendograma obtido da análise de similaridade entre as parcelas de $10 \times 40 \mathrm{~m}$, evidenciou três grupos (figura 3). O primeiro grupo é formado pela parcela 1 isoladamente; o segundo, formado pelas parcelas 2 até 11, abrange uma faixa de 100 metros após a formação pós-praia; e o terceiro (parcelas 12 a 25) atinge mais 130 metros para o interior do continente. As ligações entre parcelas do grupo 3 denotam maior similaridade florísticas quando comparadas às do grupo 2.

\section{Discussão}

Comparando os valores da densidade, área basal e diversidade encontrados neste estudo com outros realizados nas florestas de restingas brasileiras (tabela 3), foram observadas diferenças que podem ter ocorrido em função da variação de métodos empregados em cada trabalho, principalmente o critério de inclusão (DAP) ou estar refletindo as características estruturais e diversidade de cada uma das áreas, que inclui florestas que sofrem inundações em alguns períodos do ano (Sugyiama \& Mantovani 1994, César \& Monteiro 1995).

A floresta de restinga de Setiba destacou-se entre as que apresentam maior índice de diversidade e desenvolvimento diamétrico em relação aos outros estudos na costa brasileira. A elevada diversidade em Setiba também enquadra a área nos padrões de altos índices de diversidade constatados por Peixoto \& Silva
(1997) e Thomaz \& Monteiro (1997) para o Estado do Espírito Santo.

A morte de árvores é fenômeno natural e contribui com a dinâmica da vegetação em florestas tropicais (Franklin et al. 1987). Caso fossem consideradas como uma categoria específica, as árvores mortas ocupariam a $5^{\text {a }}$ colocação em VI neste estudo. A densidade de mortas obtida (67 ind.ha ${ }^{-1}$ ) aproxima-se do encontrado por Silva et al. (1994), na Ilha do Mel, SC, 66,07 ind.ha ${ }^{-1}$, ficando abaixo dos valores de outros estudos: 89,09 ind.ha ${ }^{-1}$ em Natal, RN (Trindade 1991), 104 ind.ha ${ }^{-1}$ em Setiba, ES (Fabris 1995), até 120 ind.ha $^{-1}$ em Armação de Búzios - RJ (Lobão \& Kurtz 2000). Dentre as causas indicadas por Franklin et al. (1987) que melhor explicariam a mortalidade de árvores em Setiba estariam fatores como senescência, doenças, chuva e vento.

Em florestas de restinga com solos bem drenados Myrtaceae é a principal família (VI) em diversos trechos da costa brasileira (tabela 4), com exceção de uma floresta em regeneração em Armação de Búzios, RJ. As outras famílias alternam suas posições conforme o trecho analisado, no entanto verifica-se que Sapotaceae, Annonaceae, Lauraceae, Meliaceae, Burseraceae e Leguminosae estão entre as mais importantes na maioria das florestas de restinga, corroborando com Gentry (1988) que as inclui como principais famílias neotropicais. Este autor, assim como Peixoto \& Gentry (1990), indicam Burseraceae, Lauraceae e Sapotaceae como famílias que prevalecem em solos de baixa fertilidade, como o que ocorre nas restingas (Hay \& Lacerda 1984).

Pouteria coelomatica, Myrciaria floribunda e Oxandra nitida, com os três maiores valores de VI (tabela 2), também ocorreram no trecho de floresta amostrado por Fabris (1995) no PEPCV, estando 


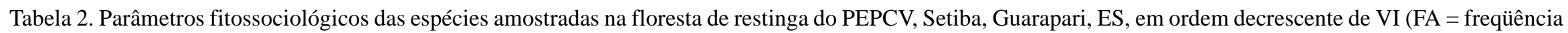

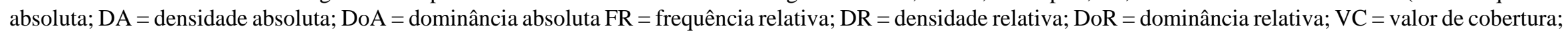
$\mathrm{VI}$ = valor de importância; Gr 1 = presente no Grupo 1 do dendograma - figura 3; Gr 2 = presente no Grupo 2 do dendograma - figura 3; Gr 3 = presente no Grupo 3 do dendograma - figura 3).

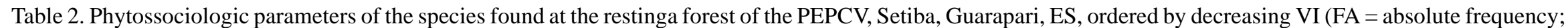
$\mathrm{DA}=$ absolute density; DoA = absolute dominance $\mathrm{FR}=$ relative frequency; $\mathrm{DR}=$ relative density; DoR = relative dominância; $\mathrm{VC}=\mathrm{value}$ of covering; $\mathrm{VI}=$ value of

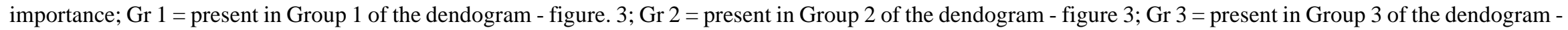
figura. 3).

\begin{tabular}{|c|c|c|c|c|c|c|c|c|c|c|c|c|}
\hline Espécies & Famílias & FA & DA & DoA & FR & $\mathrm{DR}$ & DoR & $\mathrm{VC}$ & VI & Gr 1 & Gr2 & Gr 3 \\
\hline 1. Pouteria coelomatica Rizzini & Sapotaceae & 66 & 172 & 22,48 & 5,34 & 8,17 & 8,17 & 16,33 & 21,67 & & $\mathrm{X}$ & $\mathrm{X}$ \\
\hline 2. Myrciaria floribunda (H. West. ex Willd.) O. Berg & Myrtaceae & 68 & 174 & 15,83 & 5,50 & 8,26 & 5,75 & 14,01 & 19,51 & $\mathrm{X}$ & $X$ & $\mathrm{X}$ \\
\hline 3. Oxandra nitida R.E.Fr. & Annonaceae & 52 & 179 & 17,58 & 4,20 & 8,50 & 6,39 & 14,89 & 19,09 & & $\mathrm{X}$ & $X$ \\
\hline 4. Chrysophyllum lucentifolium Cronquist & Sapotaceae & 51 & 131 & 10,46 & 4,12 & 6,22 & 3,80 & 10,02 & 14,15 & $\mathrm{X}$ & $\mathrm{X}$ & $X$ \\
\hline 5. Aspidosperma parvifolium A. DC. & Apocynaceae & 42 & 68 & 0,91 & 3,40 & 3,23 & 3,29 & 6,52 & 9,91 & & $\mathrm{X}$ & $\mathrm{X}$ \\
\hline 6. Protium heptaphyllum (Aubl.) Marchand & Burseraceae & 32 & 44 & 13,73 & 2,59 & 2,09 & 4,99 & 7,08 & 9,67 & & $\mathrm{X}$ & $X$ \\
\hline 7. Eriotheca pentaphylla (Vell. \& Schum.) A. Robyns & Bombacaceae & 29 & 63 & 0,91 & 2,34 & 2,99 & 3,31 & 6,30 & 8,64 & & & $\mathrm{X}$ \\
\hline 8. Trichilia pseudostipularis (A. Juss.) C. DC. & Meliaceae & 40 & 64 & 0,60 & 3,23 & 3,04 & 2,18 & 5,22 & 8,45 & $\mathrm{X}$ & $\mathrm{X}$ & $\mathrm{X}$ \\
\hline 9. Pseudobombax grandiflorum (Cav.) A. Robyns & Bombacaceae & 25 & 37 & 12,62 & 2,02 & 1,76 & 4,59 & 6,34 & 8,36 & $\mathrm{X}$ & $\mathrm{X}$ & $\mathrm{X}$ \\
\hline 10. Daphnopsis coriacea Taub. & Thymelaceae & 38 & 58 & 0,53 & 3,07 & 2,75 & 1,91 & 4,66 & 7,74 & $\mathrm{X}$ & $X$ & $\mathrm{X}$ \\
\hline 11. Guapira opposita (Vell.) Reitz & Nyctaginaceae & 35 & 53 & 0,64 & 2,83 & 2,52 & 2,33 & 4,84 & 7,67 & $\mathrm{X}$ & $\mathrm{X}$ & $\mathrm{X}$ \\
\hline 12. Ocotea lobbii (Meisn.) Rohwer & Lauraceae & 31 & 40 & 0,84 & 2,51 & 1,90 & 3,05 & 4,95 & 7,45 & $\mathrm{X}$ & $\mathrm{X}$ & $X$ \\
\hline 13. Zollernia glabra (Spreng.) Yakovlev & Fabaceae & 39 & 56 & 0,38 & 3,15 & 2,66 & 1,40 & 4,06 & 7,21 & & $\mathrm{X}$ & $X$ \\
\hline 14. Trichilia palens C. DC. & Meliaceae & 32 & 57 & 0,48 & 2,59 & 2,71 & 1,76 & 4,46 & 7,05 & & $\mathrm{X}$ & $\mathrm{X}$ \\
\hline 15. Campomanesia guazumifolia (Cambess.) O. Berg & Myrtaceae & 29 & 52 & 0,61 & 2,34 & 2,47 & 2,22 & 4,69 & 7,04 & $\mathrm{X}$ & $\mathrm{X}$ & $\mathrm{X}$ \\
\hline 16. Eugenia excelsa O. Berg & Myrtaceae & 29 & 59 & 0,46 & 2,34 & 2,80 & 1,67 & 4,47 & 6,81 & & $\mathrm{X}$ & $\mathrm{X}$ \\
\hline 17. Simaba cuneata A. St.-Hil. \& Tul. & Simaroubaceae & 29 & 44 & 0,61 & 2,34 & 2,09 & 2,22 & 4,31 & 6,66 & & $X$ & $\mathrm{X}$ \\
\hline 18. Gomidesia martiana O. Berg & Myrtaceae & 29 & 55 & 0,29 & 2,34 & 2,61 & 1,05 & 3,66 & 6,00 & & & $\mathrm{X}$ \\
\hline 19. Myrcia acuminantissima O. Berg & Myrtaceae & 27 & 46 & 0,33 & 2,18 & 2,18 & 1,20 & 3,39 & 5,57 & & $\mathrm{X}$ & $\mathrm{X}$ \\
\hline 20. Marlierea grandifolia O. Berg & Myrtaceae & 19 & 39 & 0,47 & 1,54 & 1,85 & 1,71 & 3,56 & 5,09 & & $\mathrm{X}$ & $\mathrm{X}$ \\
\hline 21. Rudgea reticulata Benth. & Rubiaceae & 27 & 42 & 0,25 & 2,18 & 1,99 & 0,91 & 2,91 & 5,09 & & & $\mathrm{X}$ \\
\hline 22. Annona acutiflora Mart. & Annonaceae & 30 & 37 & 0,14 & 2,43 & 1,76 & 0,50 & 2,26 & 4,69 & $\mathrm{X}$ & $\mathrm{X}$ & $\mathrm{X}$ \\
\hline 23. Pouteria sp. & Sapotaceae & 5 & 5 & 10,37 & 0,4 & 0,24 & 3,77 & 4,01 & 4,41 & & $\mathrm{X}$ & $\mathrm{X}$ \\
\hline 24. Coussapoa microcarpa (Schott) Rizzini & Cecropiaceae & 5 & 8 & 0,94 & 0,4 & 0,38 & 3,42 & 3,80 & 4,21 & & $\mathrm{X}$ & \\
\hline 25. Capparis flexuosa (L.) L. & Capparaceae & 17 & 30 & 0,38 & 1,37 & 1,42 & 1,37 & 2,80 & 4,17 & $\mathrm{X}$ & $\mathrm{X}$ & $\mathrm{X}$ \\
\hline 26. Garcinia brasiliensis Mart. & Clusiaceae & 16 & 28 & 0,42 & 1,29 & 1,33 & 1,52 & 2,85 & 4,14 & & $\mathrm{X}$ & $\mathrm{X}$ \\
\hline 27. Opuntia brasiliensis (Willd.) Haw. & Cactaceae & 19 & 25 & 0,33 & 1,54 & 1,19 & 1,20 & 2,39 & 3,93 & & $\mathrm{X}$ & $\mathrm{X}$ \\
\hline 28. Eugenia bahiensis O. Berg & Myrtaceae & 20 & 24 & 0,27 & 1,62 & 1,14 & 0,97 & 2,11 & 3,72 & & $\mathrm{X}$ & $\mathrm{X}$ \\
\hline 29. Matayba guianensis Aubl. & Sapindaceae & 21 & 23 & 0,24 & 1,7 & 1,09 & 0,86 & 1,95 & 3,65 & & $\mathrm{X}$ & $\mathrm{X}$ \\
\hline 30. Byrsonima bahiana W.R. Anderson & Malpighiaceae & 17 & 21 & 0,33 & 1,37 & 1,00 & 1,20 & 2,20 & 3,57 & & $\mathrm{X}$ & $\mathrm{X}$ \\
\hline
\end{tabular}




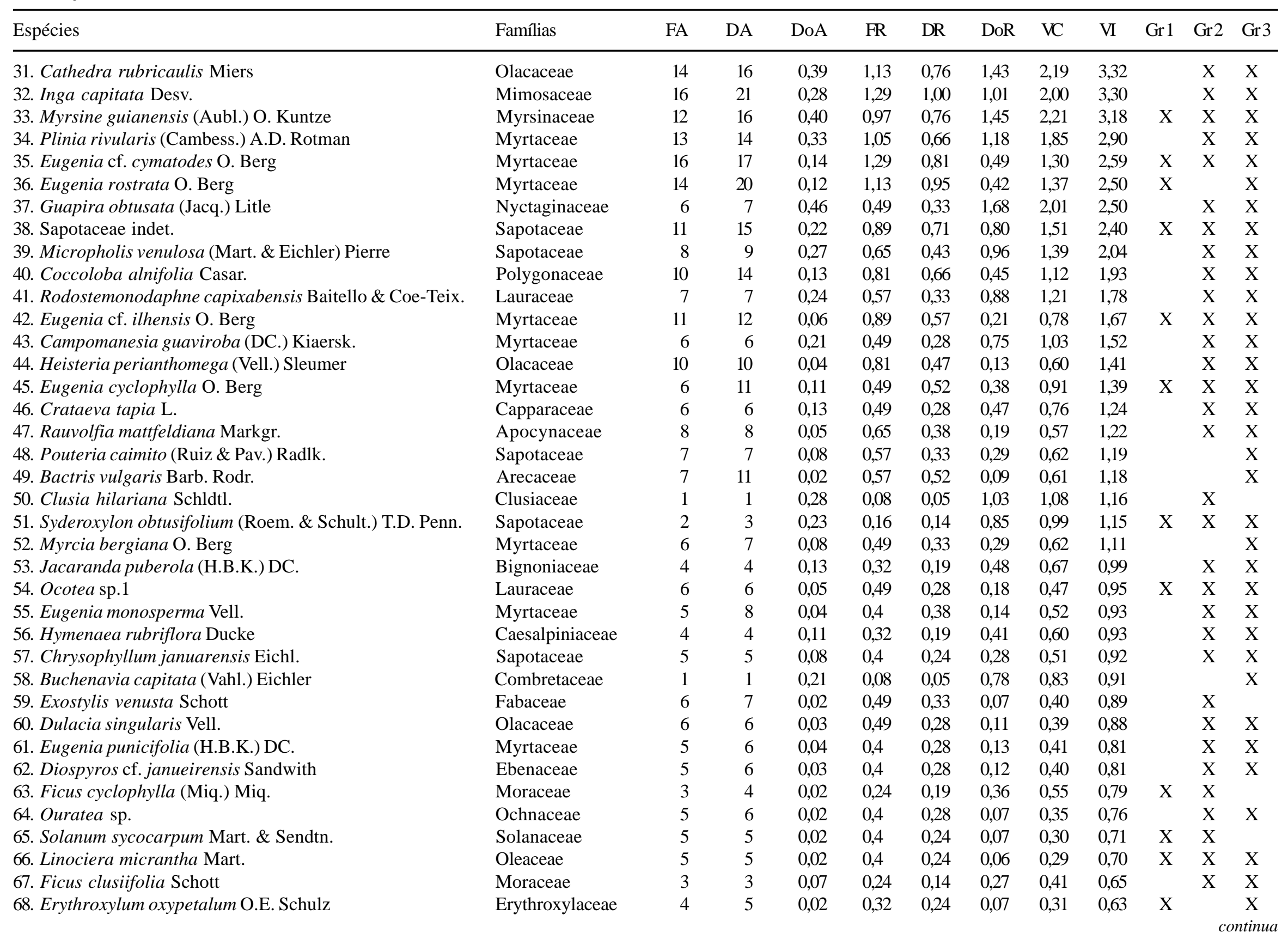




\begin{tabular}{|c|c|c|c|c|c|c|c|c|c|c|c|c|}
\hline Espécies & Famílias & FA & DA & DoA & FR & $\mathrm{DR}$ & DoR & $\mathrm{VC}$ & VI & Gr 1 & Gr 2 & Gr3 \\
\hline 69. Pera glabrata (Schott) Baill. & Euphorbiaceae & 3 & 3 & 0,07 & 0,24 & 0,14 & 0,25 & 0,39 & 0,63 & & $\mathrm{X}$ & $\mathrm{X}$ \\
\hline 70. Eugenia umbelliflora O. Berg. & Myrtaceae & 4 & 4 & 0,03 & 0,32 & 0,19 & 0,12 & 0,31 & 0,63 & & & $\mathrm{X}$ \\
\hline 71. Picramnia glazioviana Engler & Simaroubaceae & 4 & 4 & 0,03 & 0,32 & 0,19 & 0,09 & 0,28 & 0,61 & & $\mathrm{X}$ & $\mathrm{X}$ \\
\hline 72. Guarea guidonia Vahl & Meliaceae & 4 & 4 & 0,02 & 0,32 & 0,19 & 0,08 & 0,27 & 0,59 & & & $\mathrm{X}$ \\
\hline 73. Eugenia sp. & Myrtaceae & 3 & 5 & 0,03 & 0,24 & 0,24 & 0,11 & 0,35 & 0,59 & & $\mathrm{X}$ & $\mathrm{X}$ \\
\hline 74. Pouteria peduncularis (Mart. \& Eichl.) Baehni & Sapotaceae & 3 & 3 & 0,05 & 0,24 & 0,14 & 0,18 & 0,33 & 0,57 & & $\mathrm{X}$ & $\mathrm{X}$ \\
\hline 75. Ocotea sp. 2 & Lauraceae & 3 & 3 & 0,04 & 0,24 & 0,14 & 0,16 & 0,30 & 0,54 & & $\mathrm{X}$ & $\mathrm{X}$ \\
\hline 76. Erythroxylum sp. & Erythroxylaceae & 3 & 4 & 0,02 & 0,24 & 0,19 & 0,08 & 0,27 & 0,52 & & $\mathrm{X}$ & \\
\hline 77. Kielmeyera albopunctata Saddi & Clusiaceae & 2 & 3 & 0,04 & 0,16 & 0,14 & 0,16 & 0,30 & 0,46 & & & $\mathrm{X}$ \\
\hline 78. Mollinedia glabra (Spreng.) Perkins & Monimiaceae & 3 & 3 & 0,02 & 0,24 & 0,14 & 0,06 & 0,21 & 0,45 & & $\mathrm{X}$ & \\
\hline 79. Andira nitida Mart. ex Benth. & Fabaceae & 1 & 1 & 0,08 & 0,08 & 0,05 & 0,28 & 0,33 & 0,41 & & & $\mathrm{X}$ \\
\hline 80. Chlorophora tinctoria (L.) Benth. \& Hook. & Moraceae & 1 & 1 & 0,06 & 0,08 & 0,05 & 0,22 & 0,27 & 0,35 & $\mathrm{X}$ & & \\
\hline 81. Eugenia speciosa Cambess. & Myrtaceae & 2 & 2 & 0,02 & 0,16 & 0,09 & 0,05 & 0,15 & 0,31 & & $\mathrm{X}$ & $\mathrm{X}$ \\
\hline 82. Mouriri arborea Gardner & Melastomataceae & 2 & 2 & 0,01 & 0,16 & 0,09 & 0,03 & 0,13 & 0,29 & & $\mathrm{X}$ & $\mathrm{X}$ \\
\hline 83. Allophylus puberulus (A. St.-Hil.) Radlk. & Sapindaceae & 2 & 2 & 0,01 & 0,16 & 0,09 & 0,03 & 0,12 & 0,29 & & $\mathrm{X}$ & $\mathrm{X}$ \\
\hline 84. Ocotea aff. diospyrifolia (Meisn.) Mez & Lauraceae & 1 & 1 & 0,03 & 0,08 & 0,05 & 0,11 & 0,16 & 0,24 & & $\mathrm{X}$ & $\mathrm{X}$ \\
\hline 85. Ficus hirsuta Schott & Moraceae & 1 & 1 & 0,02 & 0,08 & 0,05 & 0,06 & 0,10 & 0,18 & & & $\mathrm{X}$ \\
\hline 86. Schinus terebinthifolius Raddi & Anacardiaceae & 1 & 1 & 0,01 & 0,08 & 0,05 & 0,05 & 0,10 & 0,18 & $\mathrm{X}$ & & \\
\hline 87. Cupania emarginata Cambess. & Sapindaceae & 1 & 1 & 0,01 & 0,08 & 0,05 & 0,05 & 0,09 & 0,17 & & & $\mathrm{X}$ \\
\hline 88. Amaioua guianensis A. DC. & Rubiaceae & 1 & 1 & 0,01 & 0,08 & 0,05 & 0,04 & 0,09 & 0,17 & & & $\mathrm{X}$ \\
\hline 89. Clusia spiritu-sanctensis G. Maris \& Weinberg & Clusiaceae & 1 & 1 & 0,02 & 0,08 & 0,05 & 0,02 & 0,07 & 0,15 & & & $\mathrm{X}$ \\
\hline 90. Maytenus obtusifolia Mart. & Celastraceae & 1 & 1 & 0,02 & 0,08 & 0,05 & 0,02 & 0,07 & 0,15 & & & $\mathrm{X}$ \\
\hline 91. Myrcia fallax (Rich.) DC. & Myrtaceae & 1 & 1 & 0,01 & 0,08 & 0,05 & 0,01 & 0,06 & 0,14 & & & $\mathrm{X}$ \\
\hline 92. Rauia nodosa (Engl.) Kallunki & Rutaceae & 1 & 1 & 0,01 & 0,08 & 0,05 & 0,01 & 0,05 & 0,13 & & & $\mathrm{X}$ \\
\hline
\end{tabular}




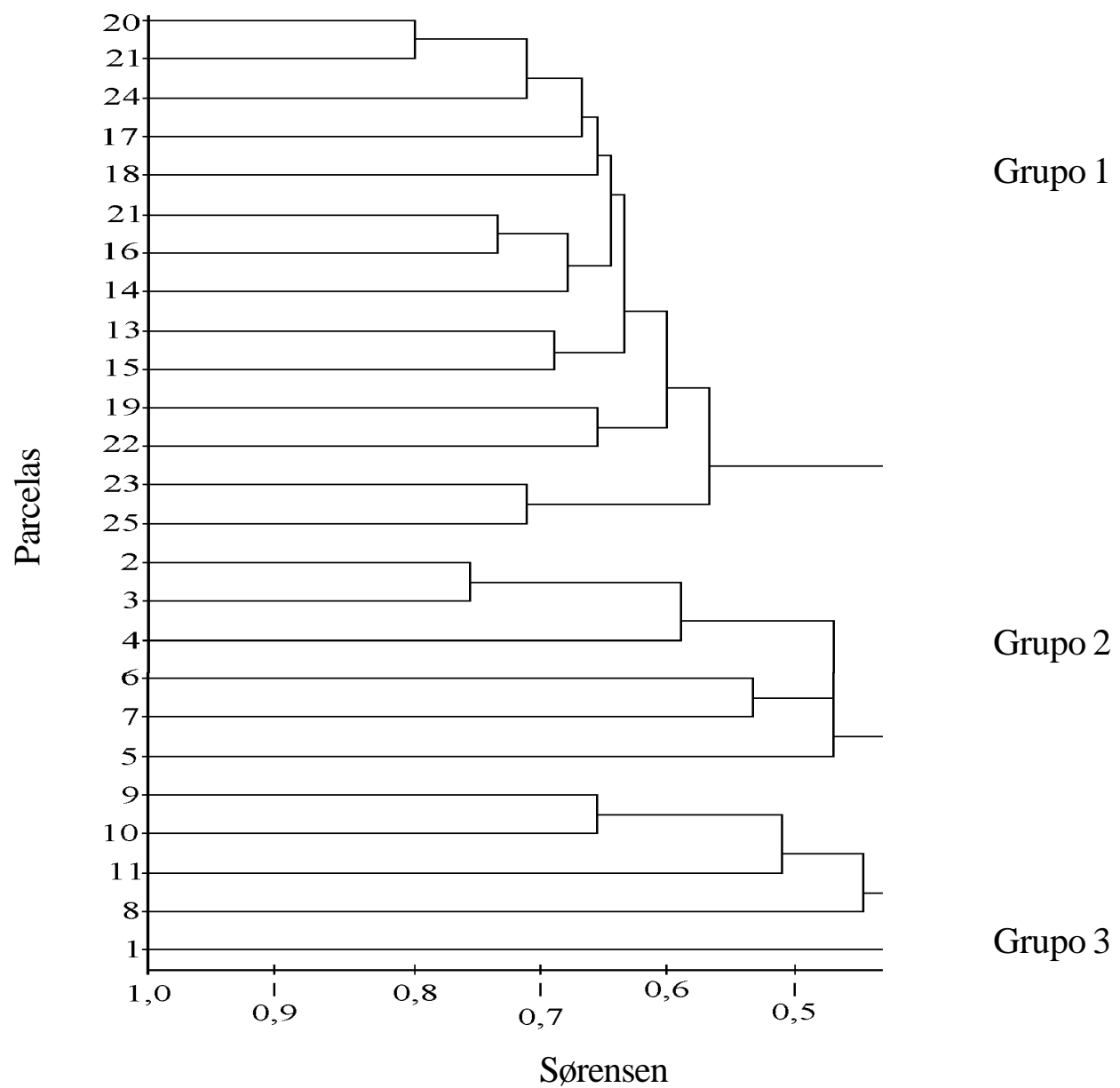

Figura 3. Dendograma de similaridade florística entre as parcelas amostradas no Parque Estadual Paulo César Vinha, Setiba, município de Guarapari (ES). Parcelas de $10 \times 40 \mathrm{~m}$.

Figure 3. Dendogram of floristic similarity among the sampled parcels in the "Paulo César Vinha" State Park, Setiba, municipally of Guarapari (ES). Parcels of $10 \times 40 \mathrm{~m}$.

P. coelomatica na primeira colocação, enquanto as demais aparecem com valores de VI menores que os encontrados neste levantamento.

As 20 espécies com maiores VI representam mais de $65 \%$ desse parâmetro (tabela 2), constituindo os componentes principais na estrutura desta comunidade. Em outros trabalhos aparecem ocupando diferentes posições de VI, com exceção de Protium heptaphyllum, que está dentre as dez espécies com maior VI em Setiba, ES (Fabris 1995), São João da Barra, RJ (Assumpção \& Nascimento 2000) e na Ilha de Algodoal, PA (Bastos 1996), além de estar entre as 20 mais importantes no Parque Estadual das Dunas, RN (Trindade 1991). Dessa maneira, poderia ser indicada como uma espécie característica das florestas de restinga da costa brasileira, com destaque no Estado do Espírito Santo, onde ocorre em diferentes localidades
(Pereira \& Zambom 1998, Pereira et al. 1998, Pereira \& Assis 2000, Pereira et al. 2000).

As espécies com um único indivíduo amostrado, consideradas raras (Martins 1979), perfazem 13\% do total analisado (tabela 2). As espécies raras neste estudo ocorrem em outras florestas de restingas com maiores densidade e VI, como Amaioua guianensis em Iguape, SP (Ramos Neto 1993), Myrcia fallax em Ubatuba, SP (César \& Monteiro 1995) e Algodoal, PA (Bastos 1996), Buchenavia capitata e Clusia hilariana em Guarapari, ES (Fabris 1995) e Schinus terebinthifolius e Maytenus obtusifolia em algumas restingas degradadas no Estado do Rio de Janeiro (Assumpção \& Nascimento 2000, Lobão \& Kurtz 2000).

No levantamento de Fabris (1995) em uma floresta na restinga de Setiba, próxima ao local desse estudo, Clusia spiritu-sanctensis também foi considerada rara, 
Tabela 3. Parâmetros estruturais e diversidade em algumas florestas de planícies costeira brasileiras, destacando o critério de inclusão e área amostral.

Table 3. Structural parameters and diversity in some Brazilian plain coastal forests, detailing the criteria of inclusion and sampled area.

\begin{tabular}{|c|c|c|c|c|c|c|}
\hline Localidade & Referência & $\begin{array}{c}\text { Densidade } \\
\text { (ind.ha }{ }^{-1} \text { ) }\end{array}$ & $\begin{array}{c}\text { Área basal } \\
\text { (ind.ha }^{-1} \text { ) }\end{array}$ & $\begin{array}{c}\mathrm{H}^{\prime} \\
\text { (nats) }\end{array}$ & $\begin{array}{c}\text { Área } \\
\text { (ha) }\end{array}$ & $\begin{array}{l}\text { DAP } \\
(\mathrm{cm})\end{array}$ \\
\hline Presente estudo & - & 2106 & 27,52 & 3,73 & 1,00 & 4,8 \\
\hline Guarapari, ES & Fabris 1995 & 3082 & 32,09 & 3,70 & 0,50 & 4,8 \\
\hline São João da Barra, RJ * & Assumpção \& Nascimento 2000 & 4222 & - & 2,81 & 0,09 & $2,5 *$ \\
\hline Armação de Búzios, RJ & Lobão \& Kurtz 2000 & 3120 & 21,82 & 2,52 & 0,10 & 2,5 \\
\hline Iguape, SP & Ramos Neto 1993 & 1993 & 30,63 & 3,37 & 0,15 & 6,3 \\
\hline Ubatuba, SP & César \& Monteiro 1995 & 1915 & 12,56 & 3,48 & 0,52 & 4,8 \\
\hline Ilha do Cardoso, SP & Sugyiama 1998 & 4652 & 27,36 & 3,09 & 0,36 & 2,5 \\
\hline Ilha do Mel, PR & Silva et al. 1994 & 2763 & 46,46 & 3,22 & 0,56 & 4,8 \\
\hline Natal, RN & Trindade 1991 & 2115 & 21,09 & 3,17 & 1,20 & 4,8 \\
\hline Maracanã, PA & Bastos 1996 & 6060 & 16,24 & 3,45 & 0,05 & 2,5 \\
\hline
\end{tabular}

* foi utilizado o diâmetro à altura do solo (DAS)

indicando, desta forma, possivelmente ser uma espécie com baixa densidade nesse tipo de ambiente. Sua distribuição geográfica está restrita aos Estados do Espírito Santo e Rio de Janeiro (Pereira \& Araujo 2000), demonstrando a importância da proteção dos ambientes onde ocorre para conservação dessa espécie.

Os grupos formados no dendograma denotam diferenças na composição florística da floresta no gradiente mar-continente (figura 3 ). A análise fitossociológica dos grupos 2 e 3 evidenciou diferença entre as 20 principais espécies, que equivalem a mais de $65 \%$ do total de VI desses grupos, apresentando oito espécies em comum, com valores e posições de VI muito diferenciadas em cada grupo (tabelas 5, 6).

Onze espécies ocorrem exclusivamente na faixa de floresta representada pelos grupos 1 e 2 (tabela 2), como Schinus terebinthifolius e Chlorophora tinctoria presentes apenas na parcela 1 , razão pela qual apresenta baixa similaridade com as demais faixas da floresta. Este trecho corresponde à transição da formação arbustiva "pós-praia" para a florestal, fenômeno verificado pelas menores médias em altura

Tabela 4. Principais famílias (ordem decrescente de VI) em trabalhos fitossociológicos desenvolvidos em florestas de restinga com solo não inundável na costa brasileira.

Table 4. Main families (arranged by importance value - decreasing VI) in phytossociologic works carried out in restinga forests in non flooded areas of the Brazilian coast.

\begin{tabular}{llllll}
\hline Presente estudo & $\begin{array}{l}\text { Guarapari, ES } \\
\text { (Fabris 1995) }\end{array}$ & $\begin{array}{l}\text { Armação de Búzios, RJ } \\
\text { (Lobão \& Kurtz 2000) }\end{array}$ & $\begin{array}{l}\text { Cananéia, SP } \\
\text { (Sugyiama 1993 } \\
\text { - Área 1) }\end{array}$ & $\begin{array}{l}\text { Natal, RN } \\
\text { (Trindade 1991) }\end{array}$ & $\begin{array}{l}\text { Maracanã, PA } \\
\text { (Bastos 1996) }\end{array}$ \\
\hline $\begin{array}{l}\text { Myrtaceae } \\
\text { Sapotaceae }\end{array}$ & Myrtaceae & Meliaceae & Myrtaceae & Myrtaceae & Myrtaceae \\
Annonaceae & Bombacaceae & Myrtaceae & Palmae & Caesalpiniaceae & Anacardiaceae \\
Bombacaceae & Leguminosae & Leguminosae & Guttiferae & Malpighiaceae & Rubiaceae \\
Meliaceae & Clusiaceae & Myrsinaceae & Theaceae & Bignoniaceae & Chrysobalanaceae \\
Apocynaceae & Simaroubaceae & Nyctaginaceae & Aquifoliaceae & Sapindaceae & Burseraceae \\
Lauraceae & Burseraceae & Erythroxylaceae & Malpighiaceae & Rubiaceae & Flacourtiaceae \\
Nyctaginaceae & Apocynaceae & Cactaceae & Leguminosae & Verbenaceae & Sapotaceae \\
Burseraceae & Lauraceae & Rhamnaceae & Cunnoniaceae & Simaroubaceae & Palmae \\
Fabaceae & Meliaceae & Capparaceae & Myrsinaceae & Sapotaceae & Annonaceae
\end{tabular}


Tabela 5. Parâmetros fitossociológicos das 20 principais espécies, em ordem decrescente de VI, do grupo 2 formado pelas parcelas 2 a 11 na análise de similaridade florística no Parque Estadual Paulo César Vinha, Setiba, município de Guarapari (ES) $(\mathrm{FR}=$ freqüência relativa; $\mathrm{DR}=$ densidade relativa; DoR = dominância relativa; VC = valor de cobertura; VI = valor de importância; * = espécies em comum aos grupos 2 e 3 ).

Table 5. Phytossociologic parameters of the 20 main species, ordered importance value - decreasing VI, belonging to group 2 formed by parcels 2 to 11 in the analysis of floristic similarity in the "Paulo César Vinha" State Park, Setiba, municipally of Guarapari (ES). (FR = relative frequency; $\mathrm{DR}=$ relative density; DoR = relative dominance; $\mathrm{VC}=\mathrm{value}$ of covering; $\mathrm{VI}=\mathrm{value}$ of importance; $*=$ species in common to groups 2 and 3 ).

\begin{tabular}{|c|c|c|c|c|c|}
\hline Espécies & FR & DR & DoR & $\mathrm{VC}$ & VI \\
\hline Oxandra nitida * & 4,27 & 19,65 & 11,76 & 31,41 & 35,68 \\
\hline Chrysophyllum lucentifolius & 4,27 & 13,22 & 6,69 & 19,92 & 24,19 \\
\hline Myrciaria floribunda * & 4,27 & 11,96 & 6,46 & 18,42 & 22,69 \\
\hline Trichilia pseudostipularis & 3,85 & 6,05 & 4,44 & 10,48 & 14,33 \\
\hline Campomanesia guazumifolia & 2,99 & 5,29 & 4,22 & 9,51 & 12,50 \\
\hline Pouteria coelomatica $*$ & 2,99 & 4,03 & 3,84 & 7,87 & 10,86 \\
\hline Coussapoa microcarpa & 2,14 & 1,01 & 7,42 & 8,43 & 10,56 \\
\hline Pouteria sp. & 1,28 & 0,50 & 8,13 & 8,64 & 9,92 \\
\hline Aspidosperma parvifolium * & 3,85 & 2,39 & 3,44 & 5,84 & 9,68 \\
\hline Pseudobombax grandiflorum * & 2,56 & 1,26 & 4,79 & 6,05 & 8,62 \\
\hline Marlierea grandifolia & 2,56 & 2,77 & 2,74 & 5,51 & 8,08 \\
\hline Protium heptaphyllum * & 2,56 & 1,39 & 4,08 & 5,47 & 8,03 \\
\hline Guapira opposita * & 2,99 & 2,90 & 1,90 & 4,79 & 7,78 \\
\hline Sapotaceae sp.1 & 2,99 & 1,51 & 1,61 & 3,12 & 6,11 \\
\hline Capparis flexuosa & 1,71 & 2,02 & 1,93 & 3,95 & 5,66 \\
\hline Myrsine guianensis & 2,14 & 1,13 & 2,22 & 3,35 & 5,49 \\
\hline Matayba guianensis & 2,56 & 1,26 & 1,03 & 2,29 & 4,86 \\
\hline Zollernia glabra * & 2,56 & 1,39 & 0,72 & 2,10 & 4,67 \\
\hline Garcinia brasiliensis & 1,71 & 1,39 & 1,54 & 2,93 & 4,64 \\
\hline Cathedra rubricaulis & 2,14 & 1,13 & 1,18 & 2,32 & 4,45 \\
\hline
\end{tabular}

(5,4 m), diâmetro $(9,9 \mathrm{~cm})$, e presença de espécies lenhosas daquela formação, como o próprio S. terebinthifolius, além de Capparis flexuosa, Syderoxylum obtusifolium e Alophyllus puberulus (Fabris et al. 1990, Pereira 1990), cujas densidade e frequiência diminuem à medida que se afasta do mar.

$\mathrm{Na}$ outra faixa (grupo 3), o número de espécies exclusivas (18) é maior (tabela 2). Oito dessas são mencionadas por Fabris (1995), que estudou uma trecho da floresta de Setiba próximo daquele ocupado pelas parcelas do grupo 3, indicando possível preferência destas espécies pela faixa de floresta afastada do mar. Alguns dos táxons exclusivos como Eriotheca pentaphylla, Gomidesia martiana e Rudgea reticulata estão entre os 20 com maior VI neste trecho da vegetação (tabela 6 ).

$\mathrm{Na}$ classificação fitofisionômica proposta por Pereira (1990) para a restinga de Setiba, a formação florestal em estudo encontra-se em uma faixa composta pelas comunidades mata de Myrtaceae e mata seca, esta última com maior altura e riqueza quando comparada com a primeira. Essa classificação está fundamentada no trabalho de Araujo \& Henriques (1984) que também reconheceram os dois tipos de florestas para o Estado do Rio de Janeiro. A fitofisionomia apresentada pela mata de Myrtaceae pode estar relacionada, no caso de Setiba, à importância de duas espécies dessa família (Campomanesia guazumifolia e Myrciaria floribunda) na faixa de floresta mais próxima ao mar (tabela 5). Essas espécies, apresentam alta densidade e caule com ritidoma desfolhante, o que favorece sua identificação neste trecho, em detrimento de outras famílias. No entanto, os resultados obtidos demonstram que Myrtaceae predomina, segundo o VI, em todo o trecho analisado, inclusive na faixa mais afastada do mar, classificada como mata seca, segundo Pereira (1990); nesta última, o número de espécies de Myrtaceae é ainda maior que nas feições florestais próximas ao mar (tabela 2). 
Tabela 6. Parâmetros fitossociológicos das 20 principais espécies, em ordem decrescente de VI, do grupo 3 formado pelas parcelas 12 a 25 na análise de similaridade florística no Parque Estadual Paulo César Vinha, Setiba, município de Guarapari (ES) $(\mathrm{FR}=$ frequiência relativa; $\mathrm{DR}=$ densidade relativa; $\mathrm{DoR}=$ dominância relativa; $\mathrm{VC}=$ valor de cobertura; $\mathrm{VI}=$ valor de importância; * = espécies em comum aos grupos 2 e 3 ).

Table 6. Phytossociologic parameters of the 20 main species, ordered by decreasing VI, belonging to group 3 formed by parcels 12 to 25 in the analysis of floristic similarity in the "Paulo César Vinha" State Park, Setiba, municipally of Guarapari (ES) $(\mathrm{FR}=$ relative frequency $; \mathrm{DR}=$ relative density $; \mathrm{DoR}=$ relative dominance $\mathrm{VC}=$ value of covering; $\mathrm{VI}=$ value of importance; $*=$ species in common to groups 2 and 3 ).

\begin{tabular}{|c|c|c|c|c|c|}
\hline Espécies & FR & DR & DoR & $\mathrm{VC}$ & VI \\
\hline Pouteria coelomatica * & 2,95 & 11,32 & 12,34 & 23,66 & 26,61 \\
\hline Eriotheca pentaphylla & 2,74 & 5,09 & 6,38 & 11,47 & 14,21 \\
\hline Myrciaria floribunda * & 2,53 & 6,31 & 5,37 & 11,67 & 14,20 \\
\hline Protium heptaphyllum * & 2,74 & 2,75 & 6,43 & 9,18 & 11,91 \\
\hline Eugenia excelsa & 2,74 & 4,53 & 2,95 & 7,47 & 10,21 \\
\hline Daphnopsis coriacea & 2,74 & 4,12 & 3,22 & 7,34 & 10,08 \\
\hline Aspidosperma parvifolium * & 2,74 & 3,96 & 3,28 & 7,25 & 9,98 \\
\hline Ocotea lobbii & 2,95 & 2,91 & 3,98 & 6,89 & 9,83 \\
\hline Trichilia palens & 2,53 & 4,12 & 2,92 & 7,05 & 9,57 \\
\hline Gomidesia martiana & 2,95 & 4,45 & 2,02 & 6,47 & 9,41 \\
\hline Simaba cuneata & 2,74 & 3,07 & 3,48 & 6,55 & 9,28 \\
\hline Pseudobombax grandiflorum * & 2,32 & 2,10 & 4,67 & 6,78 & 9,09 \\
\hline Myrcia acuminantissima & 2,95 & 3,48 & 2,02 & 5,50 & 8,44 \\
\hline Rudgea retuculata & 2,53 & 3,40 & 1,76 & 5,15 & 7,68 \\
\hline Zollernia glabra * & 2,74 & 2,99 & 1,78 & 4,77 & 7,51 \\
\hline Guapira opposita * & 2,53 & 2,26 & 2,66 & 4,92 & 7,45 \\
\hline Opuntia brasiliensis & 2,53 & 1,94 & 2,30 & 4,24 & 6,77 \\
\hline Oxandra nitida * & 1,68 & 2,02 & 1,98 & 4,01 & 5,69 \\
\hline Annona acutiflora & 2,53 & 2,34 & 0,81 & 3,16 & 5,68 \\
\hline Inga capitata & 2,32 & 1,46 & 1,62 & 3,07 & 5,39 \\
\hline
\end{tabular}

Mediante as informações sobre a composição qualiquantitativa da vegetação florestal no PEPCV e, visando uma padronização e universalização da nomenclatura das formações vegetais das restingas no Brasil, é indicado o uso do termo proposto por Silva (1998), "formação florestal não inundável", para esta comunidade florestal. $\mathrm{O}$ alcance biológico/ecológico dessa formação necessita, no entanto, de mais estudos.

A floresta de restinga de Setiba apresentou desenvolvimento diamétrico e diversidade de espécies destacados em relação a outros trechos do litoral brasileiro, embora a composição florística entre essas áreas seja semelhante, principalmente para famílias. Considerando as diferenças existentes em relação a altura dos indivíduos, a variação da densidade, inclusive com exclusão de algumas espécies no sentido marcontinente, podemos afirmar a existência de uma zonação da formação florestal para o interior do continente. Entretanto, estudos complementares sobre dinâmica populacional e ecofisiologia vegetal devem ser aplicados na tentativa de elucidar os padrões de distribuição e abundância apresentados pelas espécies desta floresta de restinga no gradiente mar-continente.

Agradecimentos - Ao Fundo de Apoio à Ciência e Tecnologia da Prefeitura Municipal de Vitória (Facitec) pela concessão da bolsa de estudo ao primeiro autor; aos especialistas de várias famílias que identificaram grande parte do material, a Dorothy Sue Dunn de Araujo pelo abstract e aos revisores deste manuscrito pelas sugestões.

\section{Referências bibliográficas}

ARAUJO, D.S.D. 1987. Restingas: Síntese dos conhecimentos para a costa sul-sudeste brasileira. In Anais do I Simpósio sobre Ecossistemas da Costa Sul e Sudeste Brasileira (S. Watanabe, coord.). Aciesp, São Paulo, v.1, p.333-347.

ARAUJO, D.S.D. 1992. Vegetation types of sandy coastal plains of tropical Brazil: a first approximation. In Coastal plant communities of Latin America (U. Seeliger, ed.). Academic Press, New York, p.337-347. 
ARAUJO, D.S.D. \& HENRIQUES, R.P.B. 1984. Análise florística das restingas do estado do Rio de Janeiro. In Restingas: Origem, estrutura e processos (L.D. Lacerda, D.S.D. Araujo, R. Cerqueira \& B. Turcq, orgs.). Universidade Federal Fluminense / CEUFF, Niterói, p.159-193.

ASSUMPÇÃO, J. \& NASCIMENTO, M.T. 2000. Estrutura e composição florística de quatro formações vegetais de restinga no complexo lagunar Grussaí/Iquipari, São João da Barra, RJ, Brasil. Acta Botanica Brasilica 14:301-315.

BASTOS, M.N.C. 1996. Caracterização das formações vegetais da restinga da Princesa, Ilha de Algodoal-Pará. Tese de Doutorado, Universidade Federal do Pará, Belém.

CÉSAR, O. \& MONTEIRO, R. 1995. Florística e fitossociologia de uma floresta de restinga em Picinguaba (Parque Estadual da Serra do Mar), Município de Ubatuba - SP. Naturalia 20:89-105.

FABRIS, L.C. 1995. Composição florística e fitossociológica de uma faixa de floresta arenosa litorânea do Parque Estadual de Setiba, Município de Guarapari, ES. Dissertação de Mestrado, Universidade Estadual Paulista, Rio Claro.

FABRIS, L.C., PEREIRA, O.J. \& ARAUJO, D.S.D. 1990. Análise fitossociológica na formação pós-praia da restinga de Setiba - Guarapari - ES. In Anais do II Simpósio de Ecossistemas da Costa Sul e Sudeste Brasileira (S. Watanabe, coord.). Aciesp, São Paulo, v.3, p.455-466.

FRANKLIN, J.F., SHUGART, H.H. \& HARMON, M.E. 1987. Tree death as an ecological process. BioScience 37:550-556.

FLEXOR, J.M., MARTIN, L., SUGUIO, K. \& DOMINGUEZ, J.M.L. 1984. Gênese dos cordões arenosos da parte central da costa brasileira. In Restingas: Origem, estrutura e processos (L.D. Lacerda, D.S.D. Araujo, R. Cerqueira \& B. Turcq, orgs.). Universidade Federal Fluminense / CEUFF, Niterói, p.35-45.

GENTRY, A. 1988. Changes in plant community diversity and floristic composition on environmental and geographical gradients. Annals of the Missouri Botanical Garden 75:1-34.

HAY, J.D. \& LACERDA, L.D. 1984. Ciclagem no ecossistema de restinga. In Restingas: Origem, estrutura e processos (L.D. Lacerda, D.S.D. Araujo, R. Cerqueira \& B. Turcq, orgs.). Universidade Federal Fluminense / CEUFF, Niterói, p.461-477.

LOBÃO, A.Q. \& KURTZ, B. 2000. Fitossociologia de um trecho de mata de restinga na Praia Gordas, município de Armação de Búzios, RJ. In Anais do V Simpósio de Ecossistemas Brasileiros (S. Watanabe, coord.). Aciesp, São Paulo, v.3, p.66-73.

MARTINS, F.R. 1979. O método de quadrantes e a fitossociologia de uma floresta residual do interior do Estado de São Paulo: Parque Estadual de Vassununga. Tese de Doutorado, Universidade de São Paulo, São Paulo.
MUELLER-DOMBOIS, D. \& ELLENBERG, H. 1974. Aims and methods of vegetation ecology. John Wiley \& Sons, New York.

PEIXOTO, A.L. \& GENTRY, A. 1990. Diversidade e composição florística de mata de tabuleiro na Reserva de Linhares (Espírito Santo, Brasil). Revista Brasileira de Botânica 13:19-25.

PEIXOTO, A.L. \& SILVA, I.M. 1997. Tabuleiro forests of Northern Espirito Santo, South-eastern Brazil. In Centres of plant diversity: a guide and strategy for their conservation (S.D. Davis, org.). IUCN Publications Unit, Cambrige, v.3, p.369-372.

PEREIRA, O.J. 1990. Caracterização fitofisionômica da restinga de Setiba - Guarapari/ES. In Anais do II Simpósio de Ecossistemas da Costa Sul e Sudeste Brasileira (S. Watanabe, coord.). Aciesp, São Paulo, v.3, p.207-219.

PEREIRA, O.J. \& ARAUJO, D.S.D. 2000. Análise florística das restingas dos Estados do Espírito Santo e Rio de Janeiro. In Ecologia de Restingas e Lagoas Costeiras (F.A. Esteves \& L.D. Lacerda, eds.). Universidade Federal do Rio de Janeiro / NUPEM, Macaé, p.25-63.

PEREIRA, O.J. \& ASSIS, A.M. 2000. Florística da restinga de Camburi. Acta Botanica Brasilica 14:99-111.

PEREIRA, O.J. \& ZAMBOM, O. 1998. Composição florística da restinga de Interlagos, Vila Velha (ES). In Anais do IV Simpósio de Ecossistemas Brasileiros (S. Watanabe, coord.). Aciesp, São Paulo, v.3, p.129-139.

PEREIRA, O.J., ASSIS, A.M. \& SOUZA, R.L.D. 1998. Vegetação da restinga de Pontal do Ipiranga, Município de Linhares (ES). In Anais do IV Simpósio de Ecossistemas Brasileiros (S. Watanabe, coord). Aciesp, São Paulo, v.3, p.117-128.

PEREIRA, O.J., BORGO, J.H., RODRIGUES, I.D. \& ASSIS, A.M. 2000. Composição florística de uma floresta de restinga no município da Serra-ES. In Anais do $\mathrm{V}$ Simpósio de Ecossistemas Brasileiros (S. Watanabe, coord.). Aciesp, São Paulo, v.3, p.74-83.

RAMOS NETO, M.B. 1993. Análise florística e estrutural de duas floresta sobre a restinga, Iguape, São Paulo. Dissertação de Mestrado, Universidade de São Paulo, São Paulo.

SHEPHERD, G.J. 1986. Fitopac 1 - Manual do usuário. Departamento de Botânica, Unicamp, Campinas.

SILVA, S.M. 1998. As formações vegetais da planície litorânea da Ilha do Mel, Paraná, Brasil: Composição florística e principais características estruturais. Tese de Doutorado, Universidade Estadual de Campinas, Campinas.

SILVA, S.M., BRITEZ, R.M., SOUZA, W.S. \& JOLY, C.A. 1994. Fitossociologia do componente arbóreo da floresta de restinga da Ilha do Mel, Paranaguá, PR. In Anais do III Simpósio de Ecossistemas da Costa Sul e Sudeste Brasileira (S. Watanabe, coord.). Aciesp, São Paulo, v.3, p.33-48. 
SUGUIO, K. \& TESSLER, M.G. 1984. Planícies de cordões litorâneos quaternários do Brasil: origem e nomenclatura. In Restingas: Origem, estrutura e processos (L.D. Lacerda, D.S.D. Araujo, R. Cerqueira \& B. Turcq, orgs.). Universidade Federal Fluminense/CEUFF, Niterói, p.15-25.

SUGYIAMA, M. 1993. Estudo de florestas na restinga da Ilha do Cardoso, Cananéia, São Paulo. Dissertação de Mestrado, Universidade de São Paulo, São Paulo.

SUGYIAMA, M. 1998. Estudo de florestas da restinga da Ilha do Cardoso, Cananéia, São Paulo. Boletim do Instituto de Botânica 11:19-159.

SUGYIAMA, M. \& MANTOVANI, W. 1994. Fitossociologia de um trecho de mata de restinga na Ilha do Cardoso, SP. In Anais do III Simpósio de Ecossistemas da Costa Sul e Sudeste Brasileira (S. Watanabe, coord.). Aciesp, São Paulo, v.3, p.49-57
THOMAZ, L.D. \& MONTEIRO, R. 1997. Composição florística da Mata Atlântica de encosta da Estação Biológica de Santa Lúcia, município de Santa TeresaES. Boletim do Museu de Biologia Mello Leitão, nova série, 7:1-48.

TRINDADE, A. 1991. Estudo florístico e fitossociológico do estrato arbustivo-arbóreo de um trecho de floresta arenícola costeira do Parque Estadual das Dunas - Natal - RN. Dissertação de mestrado, Universidade Federal Rural de Pernambuco, Recife.

WAECHTER, J.L. 1985. Aspectos ecológicos da restinga do Rio Grande do Sul, Brasil. Comunicação Científica da Pontifícia Universidade Católica do Rio Grande do Sul Série Botânica 33:49-68. 